\title{
Sarcopenia and Visceral Adiposity Are Not Independent Prognostic Markers for Extensive Disease of Small-Cell Lung Cancer: A Single-Centered Retrospective Cohort Study
}

\author{
Seigo Minami ${ }^{\mathrm{a}, \mathrm{b}}$, c, Shoichi Ihara ${ }^{\mathrm{a}, \mathrm{b}}$, Kiyoshi Komuta ${ }^{\mathrm{b}}$
}

\begin{abstract}
Background: Sarcopenia and visceral adiposity have been suggested to affect prognosis and treatment efficacy in various types of cancers. The aim of our study was to evaluate whether pretreatment sarcopenia and visceral adiposity are associated with prognosis in patients with extensive-disease small-cell lung cancer (ED-SCLC).
\end{abstract}

Methods: Between September 2007 and March 2018, 128 ED-SCLC patients received first-line and platinum-based chemotherapy at our hospital. Based on pretreatment body mass index (BMI), psoas muscle index (PMI), intramuscular adipose tissue content (IMAC) and visceral-to-subcutaneous fat ratio (VSR) at lumbar vertebra L3 level, we divided these patients into two groups, and then compared overall survival (OS) and progression-free survival (PFS). Adjusted by age, serum albumin, lactate dehydrogenase (LDH), clinical stage and performance status, we detected independent prognostic factors by multivariate Cox proportional hazard analyses.

Results: We did not find any significant differences in OS and PFS between two groups divided by BMI, PMI, IMAC and VSR. According to multivariate analyses, none of BMI, PMI, IMAC and VSR was an independent prognostic factor of OS and PFS.

Conclusions: Neither pretreatment sarcopenia nor visceral adiposity is a prognostic marker of patients with ED-SCLC treated with standard regimen of platinum-based chemotherapy.

Keywords: Sarcopenia; Visceral adiposity; Extensive disease; Smallcell lung cancer; Body mass index; Psoas muscle index; Intramus-

Manuscript submitted May 2, 2020, accepted June 4, 2020

Published online August 10, 2020

aDepartment of Respiratory Medicine, Osaka Police Hospital, 10-31 Kitayama-cho, Tennoji-ku, Osaka 543-0035, Japan

${ }^{b}$ Department of Respiratory Medicine, Daini Osaka Police Hospital, 2-6-40 Karasugatsuji, Tennoji-ku, Osaka 543-8922, Japan

${ }^{\mathrm{c} C}$ Corresponding Author: Seigo Minami, Department of Respiratory Medicine, Osaka Police Hospital, 10-31 Kitayama-cho, Tennoji-ku, Osaka-City, Osaka 543-0035, Japan. Email: seigominami@oph.gr.jp

doi: https://doi.org/10.14740/wjon1289 cular adipose tissue content; Visceral-to-subcutaneous adipose tissue area ratio

\section{Introduction}

Lung cancers are pathologically classified into small-cell lung cancer (SCLC) and non-small-cell lung cancer (NSCLC). In Japan, SCLC accounts for approximately $10 \%$ in men and $7 \%$ in women of all lung cancer cases. The incidence rates of SCLC significantly decreased in both genders probably due to the decline in smoking prevalence [1]. SCLC is then classified into two stages, limited disease (LD) or extensive disease (ED), defined by the staging system of Veterans Administration Lung Study Group [2], for the purpose of optimal selection of treatment options: curative-intent chemo-radiation therapy or palliative chemotherapy alone. Despite of highly sensitive first-line regimen of platinum-based chemotherapy, SCLC is an aggressive tumor carrying poor prognosis with early systemic metastases and acquired drug resistance. According to Japanese population-based data in 2002 - 2006, 1-, 3 - and 5-year relative survival rates among LD-SCLC were $66.2 \%, 27.2 \%$ and $21.4 \%$, but those among ED-SCLC were only $34.8 \%, 5.0 \%$ and $2.7 \%$, respectively [3].

Sarcopenia is characterized by accelerated loss of skeletal muscle quantity and quality, which leads to decreased physical performance and then adverse outcomes such as falls, frailty and death [4]. Several meta-analyses revealed that sarcopenic cancer patients were associated with postoperative complications, chemotherapy-induced toxicity, worse overall survival (OS) and disease-free survival, compared with non-sarcopenic cancer patients $[5,6]$. Regarding SCLC, there were three single-institutional and retrospective studies that had investigated association between sarcopenia and prognosis [7-9]. They all suggested that sarcopenia was an independent prognostic factor. However, these studies included various stages and treatment strategies of SCLC patients, e.g. 30.7-45.3\% of LD-SCLC and $29.1-45.3 \%$ of concurrent chemo-radiotherapy. Thus, it still remains uncertain whether sarcopenia is also a promising prognostic factor for a specific patient cohort of EDSCLC treated with standard chemotherapy. On the other hand, 
visceral adiposity also has recently been drawing attention, but still remains controversial as a prognostic factor for survival of various malignancies. Visceral adipose tissue secrets various cytokines and cytokine-like factors, which potentially enhance cancer progression. However, there were various conflicting studies, indicating negative or positive associations between visceral adiposity and cancer survival [10]. There was no study investigating association of visceral adiposity with prognosis of SCLC. Compared with these indexes, body mass index (BMI) is much simpler, more widely used, and more easily calculated only by individual weight and height. However, BMI does not reflect individual components of body weight such as fat distribution, or muscle volume and quality. Although BMI has been suggested as an independent prognostic factor for advanced NSCLC [11], it still remains controversial as a prognostic factor for SCLC.

The primary objective of our study was to investigate the prognostic significance of sarcopenia and visceral adiposity in ED-SCLC. In this study, we used BMI, psoas muscle index (PMI), intramuscular adipose tissue content (IMAC) and visceral-to-subcutaneous fat ratio (VSR) as indicators of general obesity, muscle quantity, muscle quality and visceral adiposity, respectively.

\section{Materials and Methods}

\section{Patients and study design}

This study retrospectively collected 128 patients (Supplementary Material 1, www.wjon.org). This cohort was the same as that of our previous study [12]. The inclusion criteria were as follows: 1) Cytologically or histologically SCLC; 2) The firstline, cisplatin (CDDP) or carboplatin (CBDCA)-containing chemotherapy which had been initiated between September 2007 and March 2018 at Osaka Police Hospital; 3) Clinical stage IIIB or IV in the seventh lung cancer tumor-node-metastasis (TNM) classification and staging system published by the International Union Against Cancer (UICC) [13]; 4) Abdominal plain or enhanced computed tomography (CT) scan covering L3 level within 3 months, serum albumin concentration $(\mathrm{mg} / \mathrm{dL})$ and lactate dehydrogenase $(\mathrm{LDH})(\mathrm{IU} / \mathrm{L})$ in peripheral venous blood sampling within 2 weeks before the initiation of the first-line chemotherapy; and 5) No indication for curative-intent thoracic radiotherapy. We collected the following data: sex, age, BMI, smoking habits, Eastern Cooperative Oncology Group (ECOG) performance status (PS), distant metastases, venous blood test, treatment regimens, response of the first-line chemotherapy according to the Response Evaluation Criteria in Solid Tumors (RECIST) version 1.1 [14], progression-free survival (PFS) and OS. Our definitions of response rate (RR), disease control rate (DCR), PFS and OS followed those in our previous studies $[15,16]$. The data was cut-off on December 31, 2019. Our study was approved by the Osaka Police Hospital Ethics Committee and followed the Strengthening the Reporting of Observational Studies in Epidemiology (STROBE) statement [17]. This study was conducted in compliance with the Helsinki Declaration.

\section{CT image analysis}

Using cross-sectional pretreatment CT attenuation values at the level of transverse process of L3, SYNAPSE VINCENT software (Fujifilm Medical, Tokyo, Japan) automatically identified the bilateral psoas muscle area, visceral fat area and subcutaneous fat area, and then we manually corrected them. We also manually traced the bilateral multifidus muscles areas. The definitions and the Japanese sex-specific cut-off points of PMI [18], IMAC [19] and VSR [20] followed those of our previous studies $[15,16]$.

\section{Data analyses}

The methods of descriptions and comparisons of continuous, categorical and survival data followed our previous studies [21]. Briefly, these data were shown by median value with interquartile range (IQR), frequencies, median time (months) with 95\% confidential intervals (CI) and Kaplan-Meier method, and subsequently compared by the Mann-Whitney U test, Fisher's exact test, and log-rank test, respectively. Relationships between BMI, PMI, IMAC and VSR were evaluated using Spearman's correlation analysis for continuous variables and the described by Spearman's rank correlation coefficient (rs). We used Bonferoni's method for multiple comparisons and $\mathrm{P}$ value was expressed as six-fold. Using multivariate Cox proportional hazards analyses adjusted by age ( $<75$ vs. $\geq 75$ years), serum albumin ( $\geq 3.5$ vs. $<3.5 \mathrm{~g} / \mathrm{dL})$ [22], LDH ( $\leq$ upper limit of normal range (UNL) vs. $>$ UNL) [23-25], c-stage (IIIB vs. IV) [22] and ECOG-PS (0 - 1 vs. 2 - 4) [26], we evaluated BMI, PMI, IMAC and VSR as a significant prognostic factor of PFS and OS, and then showed these results by hazard ratios (HRs) with $95 \%$ CI. The age of 75 years was selected because it is the cut-off age of the latestage medical care system for the Japanese elderly. The BMI $<18.5$ vs. $\geq 18.5$ was chosen as the nutritional cut-off level of underweight. $\mathrm{P}$ values less than 0.05 were considered statistically significant in this study. Using EZR (Saitama Medical Center, Jichi Medical University, Saitama, Japan) [27], which is a graphical user interface for $\mathrm{R}$ (The R Foundation for Statistical Computing, Vienna, Austria), we conducted all statistical analyses.

\section{Results}

We divided 128 patients into high and low BMI, PMI, IMAC and VSR. We experienced 108 deaths, and 121 progressive disease or death until the date of data cut-off. There was no significant difference in characteristics between two groups, except for age and proportion of sex and hypoalbuminemia between low and high VSR groups (Tables 1-4). We did not find any significant differences in OS and PFS between two groups according to BMI, PMI, IMAC and VSR (Figs. 1, 2). As an independent prognostic factor of OS and PFS, none of BMI, PMI, IMAC and VSR was selected by multivariate Cox hazard proportional analyses with adjustment for age, serum albumin, 
Table 1. Baseline Characteristics According to BMI

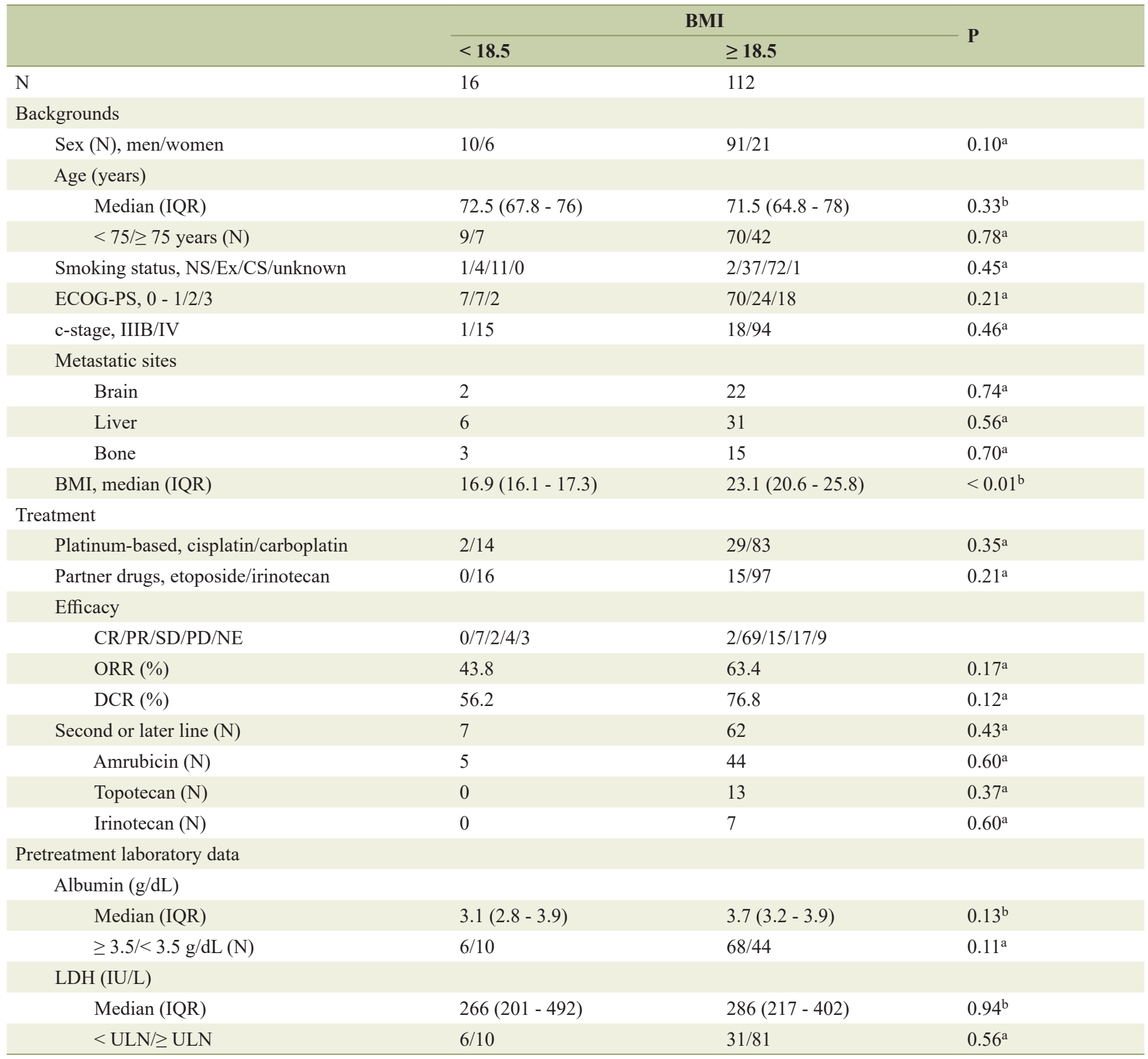

aFisher's exact test; bMann-Whitney U test. BMI: body mass index; CR: complete response; CS: current smoker; DCR: disease control rate; ECOGPS: Eastern Cooperative Oncology Group-performance status; Ex: ex-smoker; IQR: interquartile range; NE: not evaluated; NS: non-smoker; ORR: overall response rate; PD: progressive disease; PR: partial response; SD: stable disease; ULN: upper limit of normal range; LDH: lactate dehydrogenase.

LDH, c-stage and ECOG-PS (Table 5).

\section{Discussion}

Our study did not find any significant associations of sarcopenia defined by muscle quantity, PMI, with OS and PFS in ED-SCLC. These findings were contrary to the previous retrospective Korean studies, in which various sarcopenic indexes, L3 muscle index, muscle index (MI) of pectoralis muscles + high NLR, and cachexia index categorized by L3 muscle and fat indexes, were independent prognostic factors of shorter OS [7-9]. As shown in Table 6, there were differences in definitions of sarcopenia, proportion of sarcopenic patients, sex balance, clinical stage and treatment between ours and previous studies [7-9]. Our study was characterized 
Table 2. Baseline Characteristics According to PMI

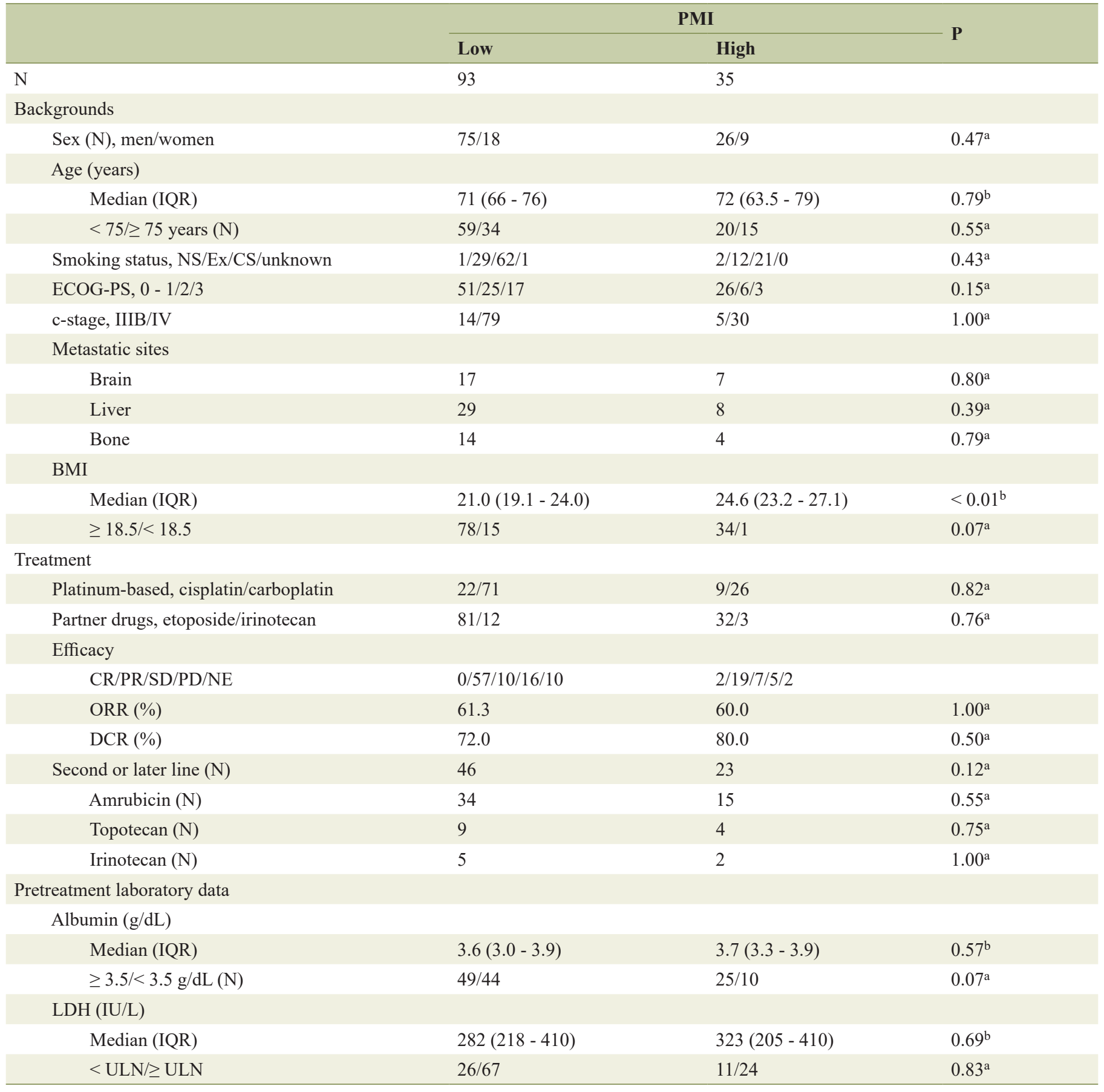

aFisher's exact test; ' Mann-Whitney U test. BMI: body mass index; PMI: psoas muscle index; CR: complete response; CS: current smoker; DCR: disease control rate; ECOG-PS: Eastern Cooperative Oncology Group-performance status; Ex: ex-smoker; IQR: interquartile range; NE: not evaluated; NS: non-smoker; ORR: overall response rate; PD: progressive disease; PR: partial response; SD: stable disease; ULN: upper limit of normal range; $\mathrm{LDH}$ : lactate dehydrogenase.

by sarcopenia sorted by PMI, higher proportion of sarcopenic patients (72.7\%), lower proportion of man (78.9\%), ED stage alone and standard chemotherapy alone. On the other hand, it is noteworthy that this was the first study investigating muscle quality indicated by IMAC in ED-SCLC. We could not detect any significant relationships of sarcopenia defined by IMAC with survivals in ED-SCLC. Therefore, our study indicated that neither muscle quantity nor quality affects prognosis of ED-SCLC.

Our study also denied association between visceral adi- 
Table 3. Baseline Characteristics According to IMAC

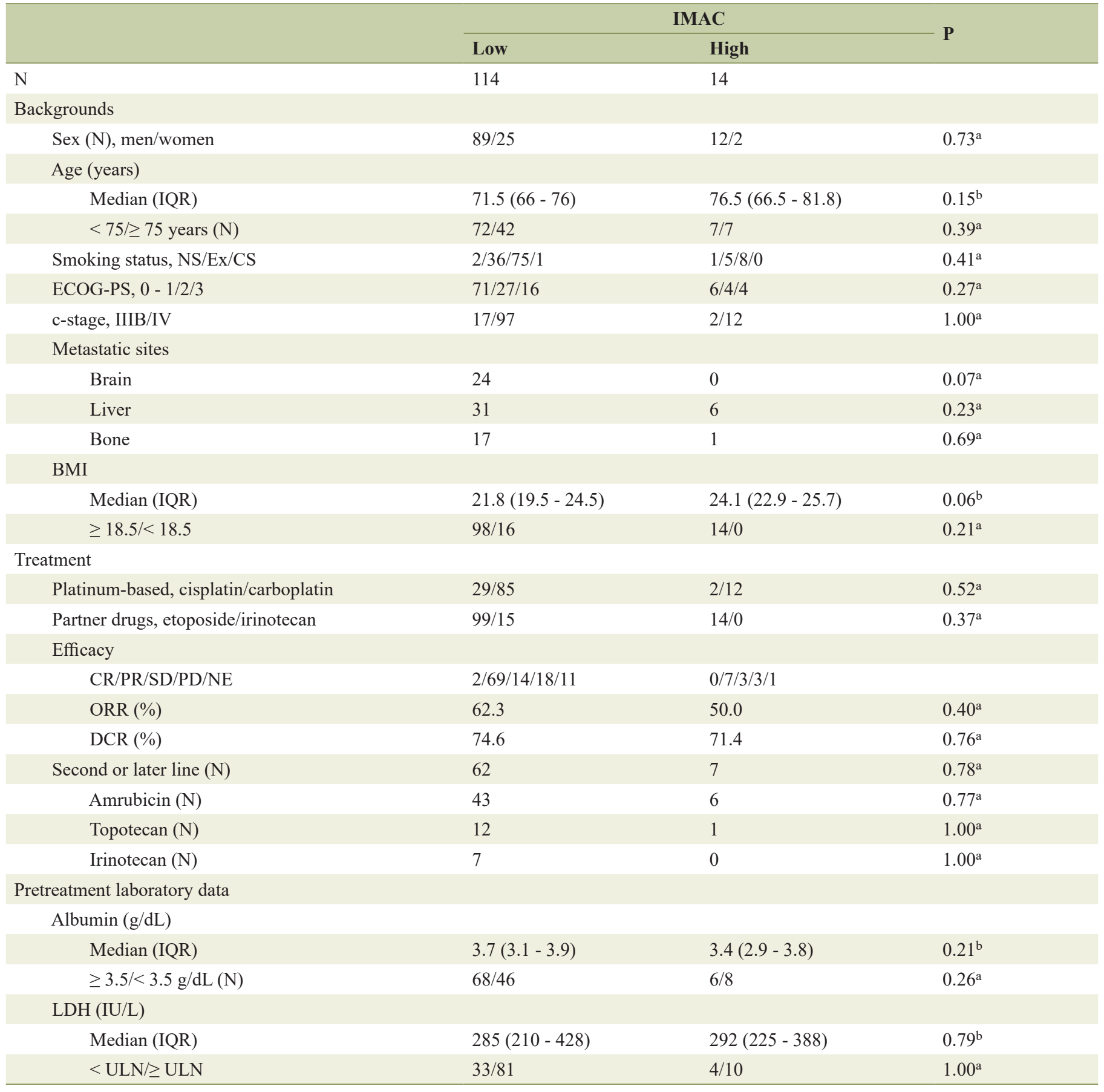

aFisher's exact test; 'bann-Whitney U test. IMAC: intramuscular adipose tissue content; BMI: body mass index; CR: complete response; CS: current smoker; DCR: disease control rate; ECOG-PS: Eastern Cooperative Oncology Group-performance status; Ex: ex-smoker; IQR: interquartile range; NE: not evaluated; NS: non-smoker; ORR: overall response rate; PD: progressive disease; PR: partial response; SD: stable disease; ULN: upper limit of normal range.

posity and prognosis of ED-SCLC. In a Korean study, cachexia scores, which categorized patients into three groups by combination of sarcopenia and/or adipopenia at L3 level, predicted prognosis in male SCLC patients. However, to our knowledge, ours was the first study that had directly focused on visceral adiposity in SCLC. Our results were consistent with our previous studies of NSCLCs treated with epidermal growth factor receptor-tyrosine kinase inhibitors [15] or with immune checkpoint inhibitor monotherapy [16]. Thus, our series of studies doubted visceral adiposity as a prognostic factor for various 
Table 4. Baseline Characteristics According to VSR

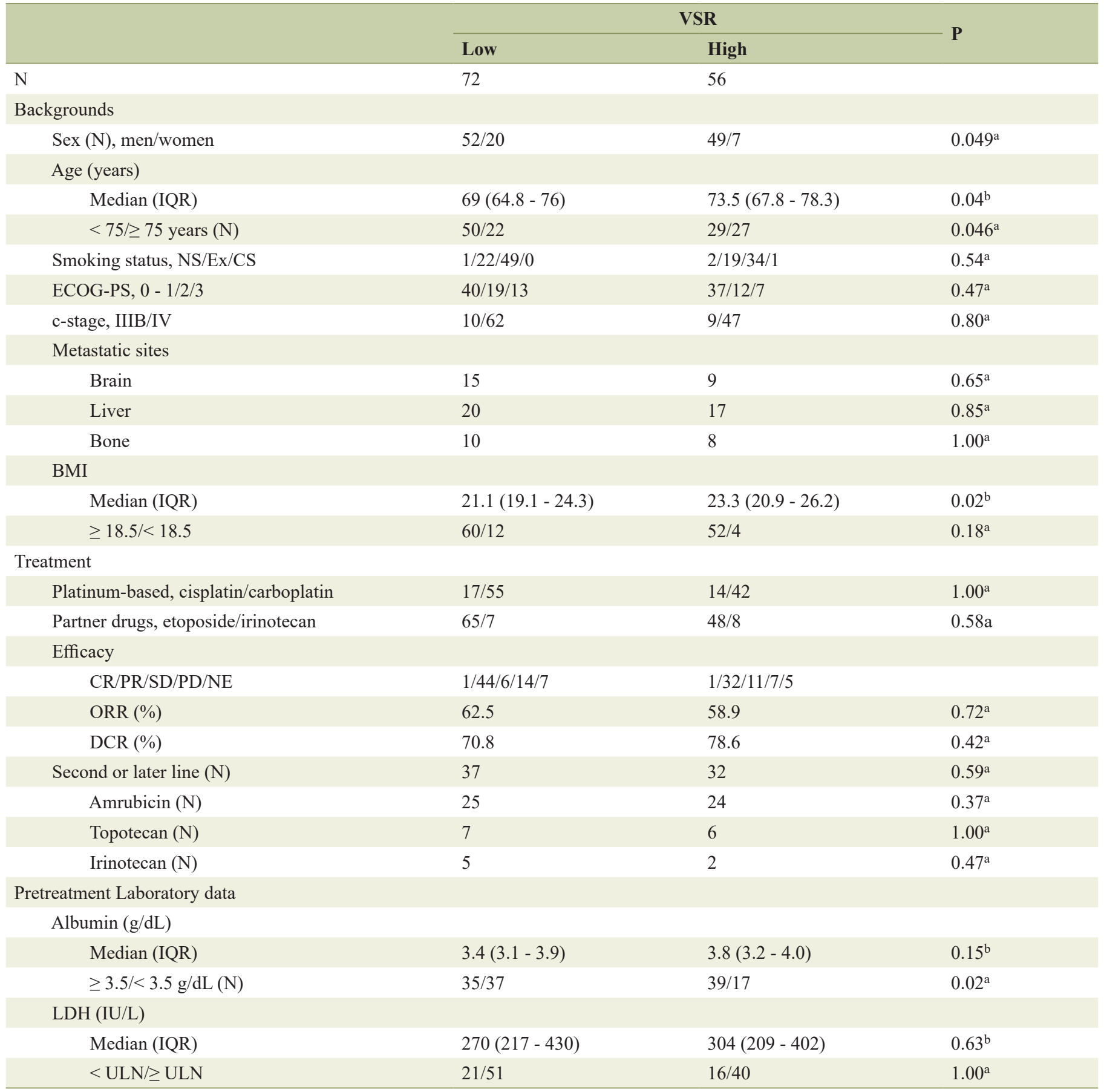

aFisher's exact test; bMann-Whitney U test. VSR: visceral-to-subcutaneous fat ratio; BMI: body mass index; CR: complete response; CS: current smoker; DCR: disease control rate; ECOG-PS: Eastern Cooperative Oncology Group-performance status; Ex: ex-smoker; IQR: interquartile range; NE: not evaluated; NS: non-smoker; ORR: overall response rate; PD: progressive disease; PR: partial response; SD: stable disease; ULN: upper limit of normal range; LDH: lactate dehydrogenase.

types of lung cancer.

Interestingly, our study also failed to detect BMI as a prognostic factor for ED-SCLC. Previous studies evaluating BMI and prognosis of SCLC were various in cut-off points of BMI, clinical stage, treatment and analytical methods [7-9, 28-31]
(Table 7). Most studies failed to demonstrate significant association of BMI with survival prognosis of SCLC [7, 8, 2830]. Our study was consistent with these previous studies. In multivariate analyses of a pooled analysis of international lung cancer consortium, none of underweight, overweight, obese at 

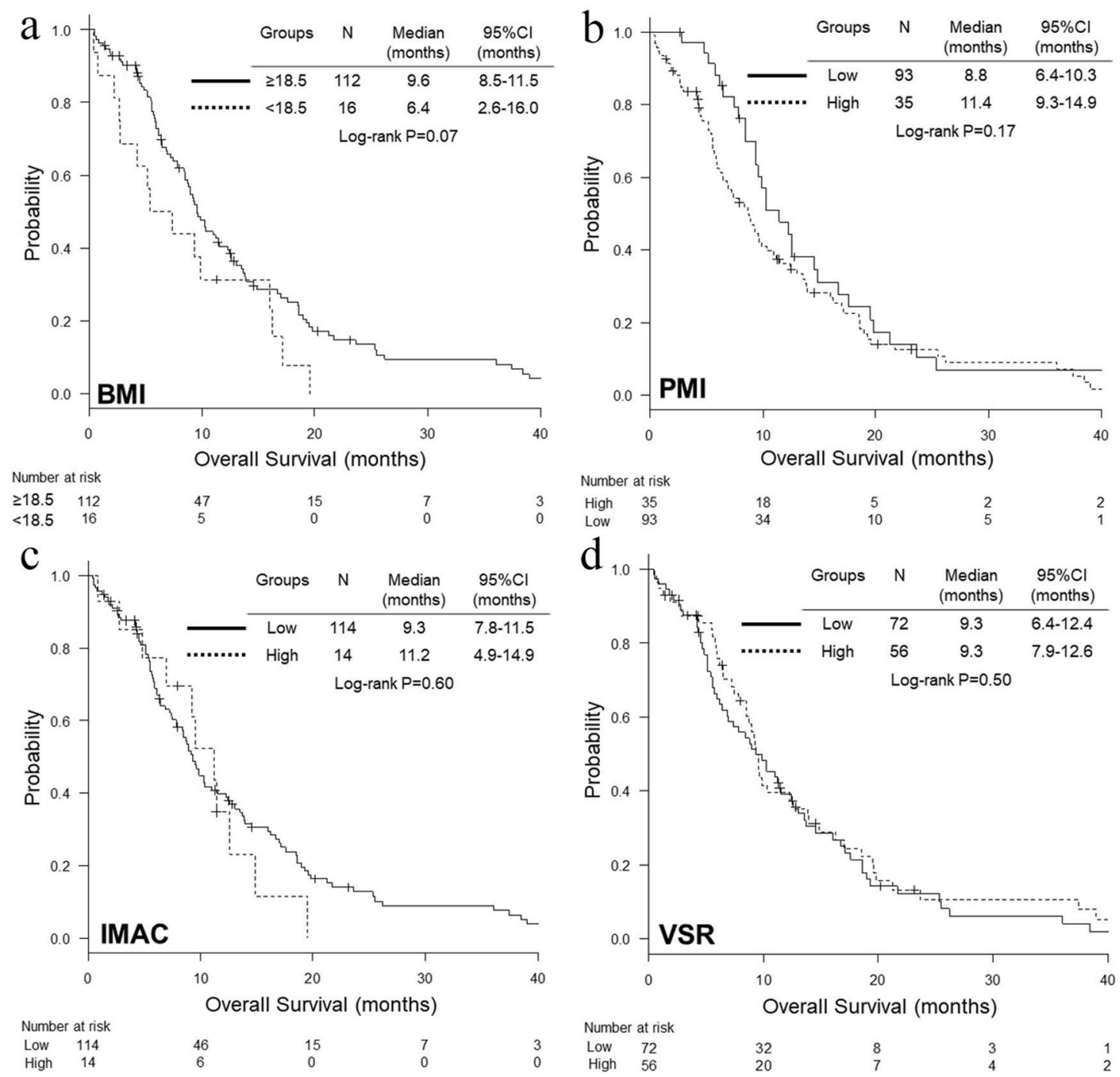

Figure 1. Kaplan-Meier curves of overall survival according to BMI (a), PMI (b), IMAC (c) and VSR (d). BMI: body mass index; PMI: psoas muscle index; IMAC: intramuscular adipose tissue content; VSR: visceral-to-subcutaneous fat ratio.

diagnosis, and decreased change in BMI from young adult age (18 - 25 years) to diagnosis was an independent prognostic factor for OS of SCLC. However, in NSCLC, among these BMIrelated variables, underweight and decreased BMI change were significantly associated with poorer OS, while overweight and obese were with improved OS [31]. We previously detected significant association of low BMI $\left(<18.5 \mathrm{~kg} / \mathrm{m}^{2}\right)$ with shorter PFS and OS in epidermal growth factor receptor (EGFR)-mutant NSCLC patients treated with EGFR-tyrosine kinase inhibitors (TKIs) [15]. Thus, there may be a difference in relations of BMI with survival between NSCLC and SCLC.

Some limitations existed in this study. First, our small sample size and single-centered retrospective study design are not enough for our results to be generalized for all EDSCLC patients. We included imbalance and small number of group patients especially in BMI and IMAC. We might miss unmeasured confounding variables, such as, nutritional status of eating or body weight change. However, our study currently denies patient selection for chemotherapy according to sarcopenia and visceral adiposity. Second, our patients had received conventional chemotherapy of platinum doublet regimen before atezolizumab with carboplatin and etoposide was approved by Japanese medical insurance in August 2020. Our study did not reflect the new era of combination immunotherapy for ED-SCLC. Further studies will be warranted for combination immunotherapy for ED-SCLC. Third, our definitions of sarcopenia and visceral adiposity were not standard, but convenient. For cancer patients, pretreatment staging CT scans are common. CT-determined definition of sarcopenia is much easier to use than standard and authorized diagnostic definitions requiring multiple steps $[4,32]$. The standard definition of visceral adiposity remains indefinite. CT-defined visceral adiposity is more reliable than conventional method of waist circumference measurement. 

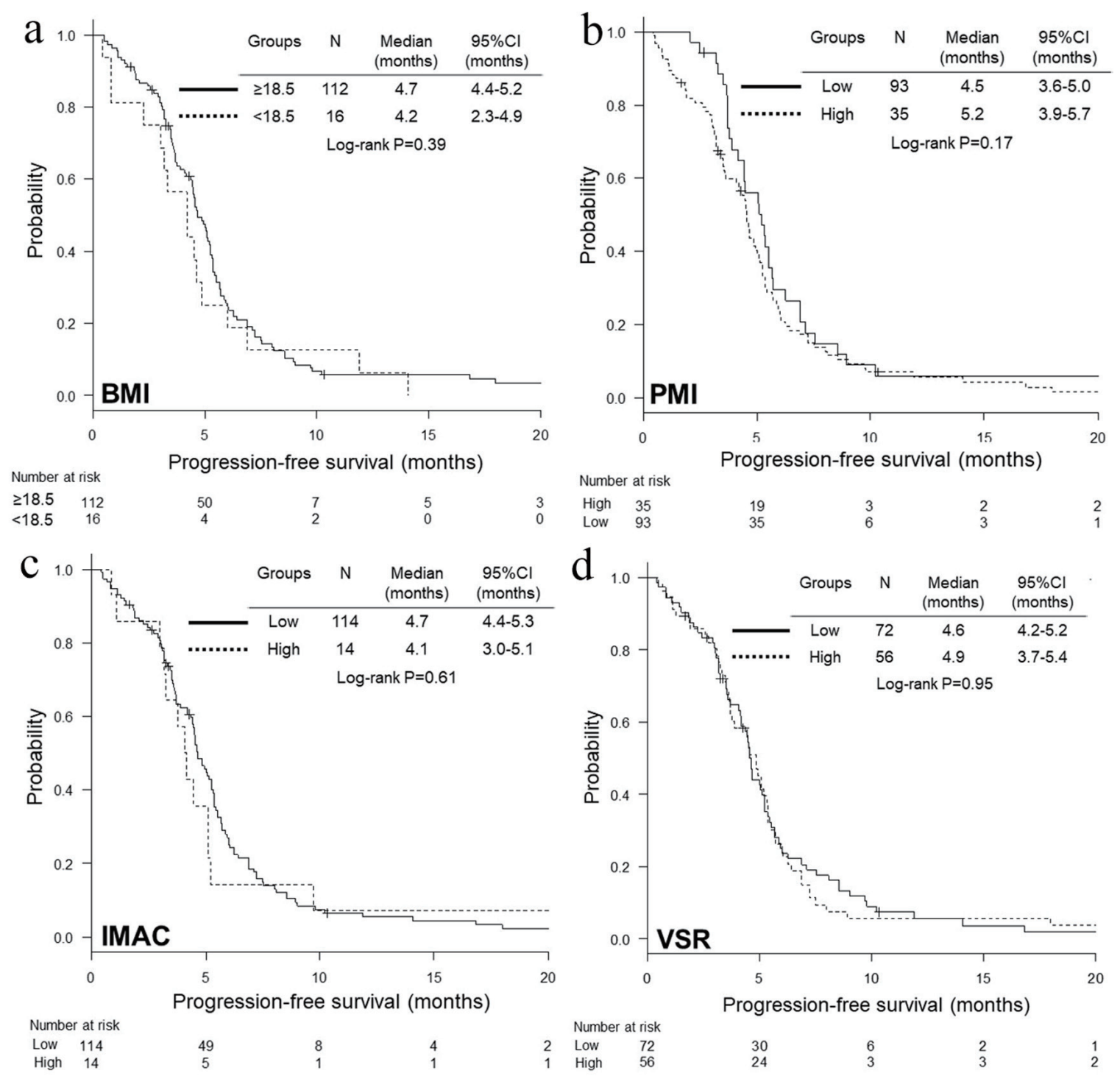

Figure 2. Kaplan-Meier curves of progression-free survival according to BMI (a), PMI (b), IMAC (c) and VSR (d). BMI: body mass index; PMI: psoas muscle index; IMAC: intramuscular adipose tissue content; VSR: visceral-to-subcutaneous fat ratio.

\section{Conclusions}

Neither pretreatment sarcopenia nor visceral adiposity is a prognostic marker of patients with ED-SCLC treated with standard regimen of platinum-based chemotherapy.

\section{Supplementary Material}

Suppl 1. Patient Selection.

\section{Acknowledgments}

We are grateful to Takeshi Nakatani, Kanako Nishimatsu,
Hideyasu Okada, Kazuki Hashimoto, Akihiro Tsukaguchi and Nao Shoshihara at the Department of Respiratory Medicine, Osaka Police Hospital, and Tsunehiro Tanaka, Kensuke Kanaoka at the Department of Respiratory Medicine, Daini Osaka Police Hospital for their medical records, diagnosis, treatment and care of their patients.

\section{Financial Disclosure}

None to declare.

\section{Conflict of Interest}

None to declare. 
Table 5. Adjusted Hazard Ratios of Indexes of Sarcopenia and Visceral Obesity for Overall Survival and Progression-Free Survival

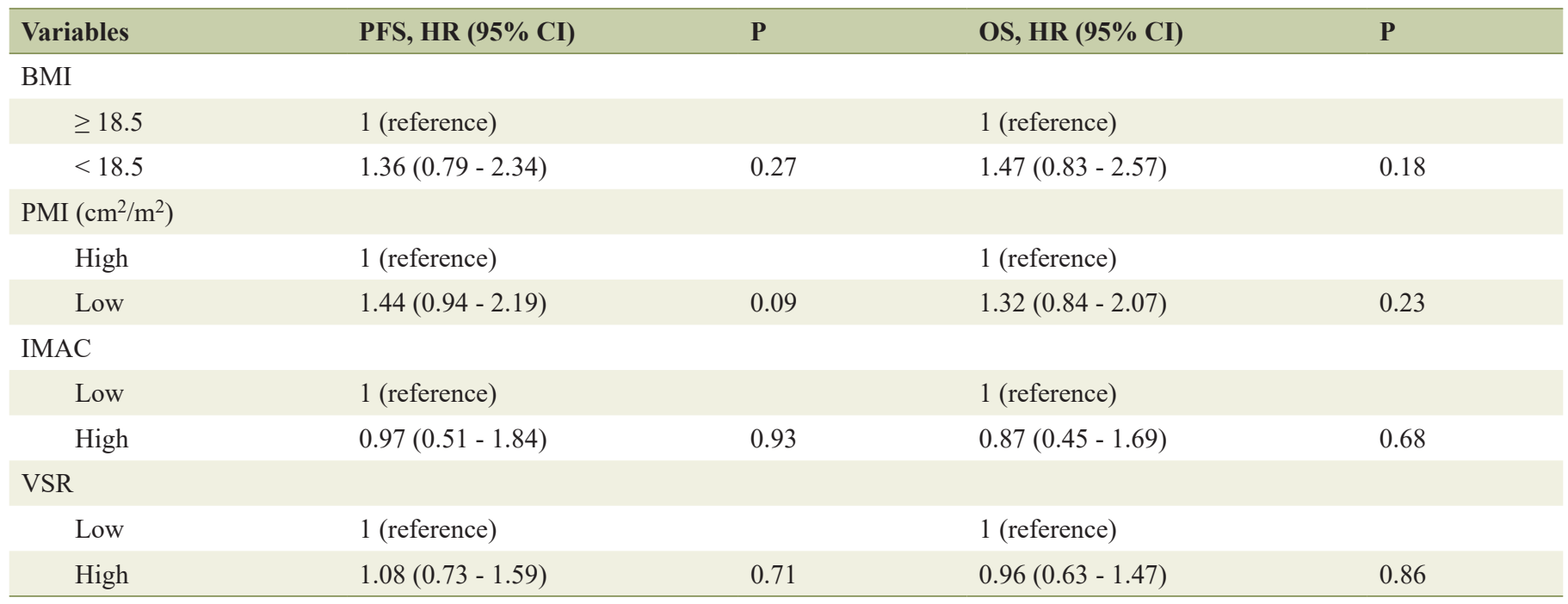

Multivariate adjustment for age (<75 vs. 575 years), serum albumin ( $\geq 3.5$ vs. $<3.5 \mathrm{~g} / \mathrm{dL}$ ), LDH ( $\leq$ UNL vs. $>$ UNL), C-stage (IIIB vs. IV) and ECOGPS (0 - 1 vs. 2 - 4). BMI: body mass index; Cl: confidence interval; ECOG-PS: Eastern Cooperative Oncology Group-performance status; HR: hazard ratio; IMAC: intramuscular adipose tissue content; LDH: lactate dehydrogenase; PMI: psoas muscle index; UNL: upper limit of normal range; VSR: visceral-to-subcutaneous fat ratio; PFS: progression-free survival; OS: overall survival.

\section{Informed Consent}

Not applicable.

\section{Author Contributions}

SM designed, performed the statistical analysis of the data, and drafted the manuscript. All authors were involved in the conceptual design, review of the draft, and approved the final manuscript. KK supervised all aspects of the study.

\section{Data Availability}

The authors declare that data supporting the findings of this study are available within the article.

Table 6. Review of Studies That Evaluated CT-Determined Sarcopenia in SCLC

\begin{tabular}{|c|c|c|c|c|}
\hline Author (year) & Patients & $\begin{array}{l}\text { Sarcopenia } \\
\text { definitions }\end{array}$ & $\begin{array}{l}\text { Survival comparison; sar- } \\
\text { copenia vs. non-sarcopenia }\end{array}$ & Multivariate analyses \\
\hline $\begin{array}{l}\text { Kim et al } \\
(2015)[7]\end{array}$ & $\begin{array}{l}149 \text { SCLC; male }(85.2 \%), \\
\text { ED }(67.8 \%) \text {, chemo alone } \\
(48.3 \%) \text {, CRT }(29.5 \%), \\
\text { BSC alone }(20.8 \%)\end{array}$ & $\begin{array}{l}\text { L3MI, sarcopenia } \\
(79.2 \%)\end{array}$ & $\begin{array}{l}\text { OS, } 8.6 \text { vs. } 16.8 \\
\text { months, } \mathrm{P}=0.031\end{array}$ & $\begin{array}{l}\text { Non-sarcopenia vs. sarcopenia; OS, } \\
\text { HR } 1.68,95 \% \text { CI } 1.04-2.72, \mathrm{P}=0.034\end{array}$ \\
\hline $\begin{array}{l}\text { Go et al } \\
\text { (2016) [8] }\end{array}$ & $\begin{array}{l}117 \text { male SCLC; ED } \\
(54.7 \%), \text { CRT }(45.3 \%)\end{array}$ & $\begin{array}{l}\text { SMI of pectoralis } \\
\text { muscles, sarcopenia } \\
(24.8 \%)\end{array}$ & $\begin{array}{l}\text { PFS, } 6.0 \text { vs. } 7.5 \text { months, } \\
P=0.009 ; \text { OS, } 10.5 \text { vs. } \\
13.5 \text { months, } P=0.052\end{array}$ & $\begin{array}{l}\text { Sarcopenia with low NLR + non- } \\
\text { sarcopenia vs. sarcopenic with high } \\
\text { NLR; PFS, HR } 3.805,95 \% \text { CI } 1.774 \\
\text { - } 8.158, \mathrm{P}=0.001 ; \text { OS, HR } 2.230 \\
95 \% \text { CI } 1.048-4.743, \mathrm{P}=0.037\end{array}$ \\
\hline $\begin{array}{l}\text { Kim et al } \\
(2017)[9]\end{array}$ & $\begin{array}{l}127 \text { male SCLC; ED } \\
(69.3 \%) \text {, chemo alone } \\
(48.0 \%) \text {, CRT }(29.1 \%), \\
\text { BSC alone }(21.3 \%)\end{array}$ & $\begin{array}{l}\text { CS defined by } \\
\text { L3MI and L3FI; } \\
\text { CS0 }(13.4 \%), \text { CS1 } \\
(74.8 \%), \text { CS2 }(11.8 \%)\end{array}$ & $\begin{array}{l}\text { OS, } 5.0(\mathrm{CS} 2) \text { vs. } 8.9 \\
(\mathrm{CS} 1) \text { vs. } 18.3 \text { months } \\
(\mathrm{CS} 0), \mathrm{P}=0.007\end{array}$ & $\begin{array}{l}\text { CS0 vs. CS1, OS, HR } 1.99,95 \% \\
\text { CI } 1.05-3.78, \mathrm{P}=0.036 ; \text { CS0 } \\
\text { vs. CS2, OS, HR } 2.59,95 \% \\
\text { CI } 1.14-5.89, \mathrm{P}=0.023\end{array}$ \\
\hline
\end{tabular}

BSC: best supportive care; chemo: chemotherapy; Cl: confidence interval; CRT: chemo-radiotherapy; CS: cachexia score; ED: extensive disease; FI: fat index; HR: hazard ratio; LD: limited disease; MI: muscle index; NLR: neutrophil-to-lymphocyte ratio; OS: overall survival; PFS: progression-free survival; PMI: psoas muscle index; SCLC: small-cell lung cancer; SMI: skeletal muscle index; CT: computed tomography. 
Table 7. Review of Studies That Evaluated BMI in Untreated SCLC

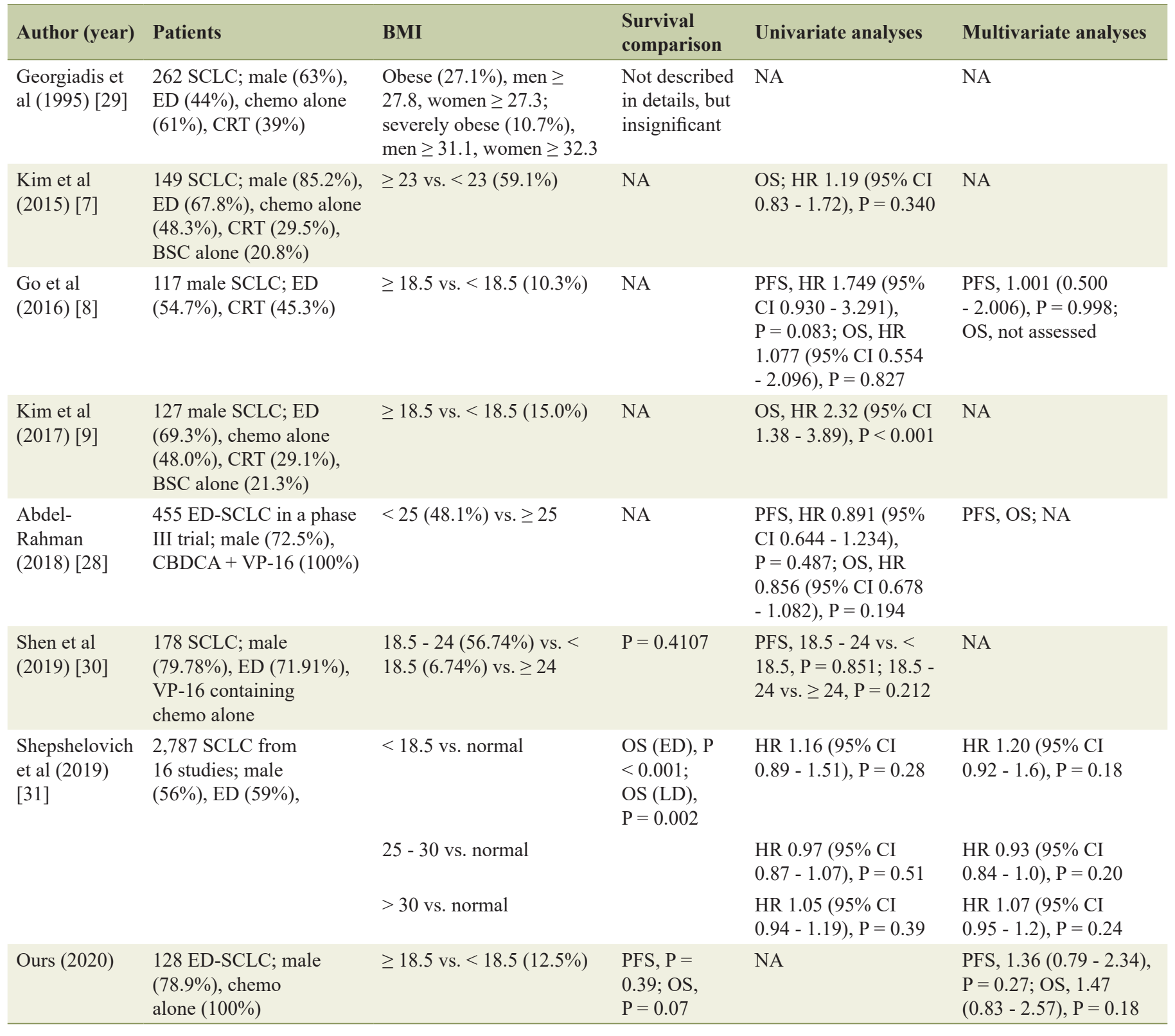

BSC: best supportive care; CBDCA: carboplatin; Chemo: chemotherapy; CI: confidence interval; CRT: chemo-radiotherapy; ED: extensive disease; LD: limited disease; HR: hazard ratio; NA: not accessed; OS: overall survival; PFS: progression-free survival; SCLC: small-cell lung cancer; VP-16: etoposide; BMI: body mass index.

\section{References}

1. Kinoshita FL, Ito Y, Nakayama T. Trends in lung cancer incidence rates by histological type in 1975-2008: a population-based study in Osaka, Japan. J Epidemiol. 2016;26(11):579-586.

2. Green RA, Humphrey E, Close H, Patno ME. Alkylating agents in bronchogenic carcinoma. Am J Med. 1969;46(4):516-525.

3. Oze I, Ito H, Nishino Y, Hattori M, Nakayama T, Miyashiro I, Matsuo K, et al. Trends in small-cell lung cancer survival in 1993-2006 based on population-based cancer registry data in Japan. J Epidemiol. 2019;29(9):347-353.

4. Cruz-Jentoft AJ, Bahat G, Bauer J, Boirie Y, Bruyere O, Cederholm T, Cooper C, et al. Sarcopenia: revised European consensus on definition and diagnosis. Age Ageing. 2019;48(1):16-31.

5. Pamoukdjian F, Bouillet T, Levy V, Soussan M, Zelek L, Paillaud E. Prevalence and predictive value of pre-therapeutic sarcopenia in cancer patients: A systematic review. Clin Nutr. 2018;37(4):1101-1113.

6. Shachar SS, Williams GR, Muss HB, Nishijima TF. Prog- 
nostic value of sarcopenia in adults with solid tumours: A meta-analysis and systematic review. Eur J Cancer. 2016;57:58-67.

7. Kim EY, Kim YS, Park I, Ahn HK, Cho EK, Jeong YM. Prognostic Significance of CT-Determined Sarcopenia in Patients with Small-Cell Lung Cancer. J Thorac Oncol. 2015;10(12):1795-1799.

8. Go SI, Park MJ, Song HN, Kang MH, Park HJ, Jeon $\mathrm{KN}$, Kim SH, et al. Sarcopenia and inflammation are independent predictors of survival in male patients newly diagnosed with small cell lung cancer. Support Care Cancer. 2016;24(5):2075-2084.

9. Kim EY, Lee HY, Kim YS, Park I, Ahn HK, Cho EK, Jeong YM, et al. Prognostic significance of cachexia score assessed by $\mathrm{CT}$ in male patients with small cell lung cancer. Eur J Cancer Care (Engl). 2018;27(1):e12695.

10. Xiao J, Mazurak VC, Olobatuyi TA, Caan BJ, Prado CM. Visceral adiposity and cancer survival: a review of imaging studies. Eur J Cancer Care (Engl). 2018;27(2):e12611.

11. Mandrekar SJ, Schild SE, Hillman SL, Allen KL, Marks RS, Mailliard JA, Krook JE, et al. A prognostic model for advanced stage nonsmall cell lung cancer. Pooled analysis of North Central Cancer Treatment Group trials. Cancer. 2006;107(4):781-792.

12. Minami S, Ihara S, Komuta K. Gustave Roussy Immune Score and Royal Marsden Hospital Prognostic Score are prognostic markers for extensive disease of small cell lung cancer. World J Oncol. 2020;11(3):98-105.

13. Rami-Porta R, Crowley JJ, Goldstraw P. The revised TNM staging system for lung cancer. Ann Thorac Cardiovasc Surg. 2009;15(1):4-9.

14. Eisenhauer EA, Therasse P, Bogaerts J, Schwartz LH, Sargent D, Ford R, Dancey J, et al. New response evaluation criteria in solid tumours: revised RECIST guideline (version 1.1). Eur J Cancer. 2009;45(2):228-247.

15. Minami S, Ihara S, Nishimatsu K, Komuta K. Low body mass index is an independent prognostic factor in patients with non-small cell lung cancer treated with epidermal growth factor receptor tyrosine kinase inhibitor. World J Oncol. 2019;10(6):187-198.

16. Minami S, Ihara S, Tanaka T, Komuta K. Sarcopenia and visceral adiposity did not affect efficacy of immunecheckpoint inhibitor monotherapy for pretreated patients with advanced non-small cell lung cancer. World J Oncol. 2020;11(1):9-22.

17. von Elm E, Altman DG, Egger M, Pocock SJ, Gotzsche PC, Vandenbroucke JP, Initiative S. The Strengthening the Reporting of Observational Studies in Epidemiology (STROBE) Statement: guidelines for reporting observational studies. Int J Surg. 2014;12(12):1495-1499.

18. Hamaguchi Y, Kaido T, Okumura S, Kobayashi A, Hammad A, Tamai Y, Inagaki N, et al. Proposal for new diagnostic criteria for low skeletal muscle mass based on computed tomography imaging in Asian adults. Nutrition. 2016;32(11-12):1200-1205.

19. Hamaguchi Y, Kaido T, Okumura S, Kobayashi A, Shirai $\mathrm{H}$, Yagi S, Kamo N, et al. Impact of Skeletal Muscle Mass Index, Intramuscular Adipose Tissue Content, and Visceral to Subcutaneous Adipose Tissue Area Ratio on
Early Mortality of Living Donor Liver Transplantation. Transplantation. 2017;101(3):565-574.

20. Fujiwara N, Nakagawa H, Kudo Y, Tateishi R, Taguri M, Watadani T, Nakagomi R, et al. Sarcopenia, intramuscular fat deposition, and visceral adiposity independently predict the outcomes of hepatocellular carcinoma. J Hepatol. 2015;63(1):131-140.

21. Minami S, Ihara S, Ikuta S, Komuta K. Gustave Roussy Immune Score and Royal Marsden Hospital Prognostic Score are biomarkers of immune-checkpoint inhibitor for nonsmall cell lung cancer. World J Oncol. 2019;10(2):90-100.

22. Shirasawa M, Fukui T, Kusuhara S, Hiyoshi Y, Ishihara M, Kasajima M, Nakahara Y, et al. Prognostic significance of the 8th edition of the TNM classification for patients with extensive disease small cell lung cancer. Cancer Manag Res. 2018;10:6039-6047.

23. Hermes A, Gatzemeier U, Waschki B, Reck M. Lactate dehydrogenase as prognostic factor in limited and extensive disease stage small cell lung cancer - a retrospective single institution analysis. Respir Med. 2010;104(12):1937-1942.

24. Quoix E, Purohit A, Faller-Beau M, Moreau L, Oster JP, Pauli G. Comparative prognostic value of lactate dehydrogenase and neuron-specific enolase in small-cell lung cancer patients treated with platinum-based chemotherapy. Lung Cancer. 2000;30(2):127-134.

25. Shirasawa M, Fukui T, Kusuhara S, Harada S, Nishinarita $\mathrm{N}$, Hiyoshi Y, Ishihara M, et al. Prognostic differences between oligometastatic and polymetastatic extensive diseasesmall cell lung cancer. PLoS One. 2019;14(4):e0214599.

26. Suzuki R, Lin SH, Wei X, Allen PK, Welsh JW, Byers LA, Komaki R. Prognostic significance of pretreatment total lymphocyte count and neutrophil-to-lymphocyte ratio in extensive-stage small-cell lung cancer. Radiother Oncol. 2018;126(3):499-505.

27. Kanda Y. Investigation of the freely available easy-touse software 'EZR' for medical statistics. Bone Marrow Transplant. 2013;48(3):452-458.

28. Abdel-Rahman O. Impact of baseline characteristics on extensive-stage SCLC patients treated with etoposide/ carboplatin: A secondary analysis of a phase III study. Clin Respir J. 2018;12(10):2519-2524.

29. Georgiadis MS, Steinberg SM, Hankins LA, Ihde DC, Johnson BE. Obesity and therapy-related toxicity in patients treated for small-cell lung cancer. J Natl Cancer Inst. 1995;87(5):361-366.

30. Shen XB, Zhang YX, Wang W, Pan YY. The Hemoglobin, Albumin, Lymphocyte, and Platelet (HALP) score in patients with small cell lung cancer before first-line treatment with etoposide and progression-free survival. Med Sci Monit. 2019;25:5630-5639.

31. Shepshelovich D, Xu W, Lu L, Fares A, Yang P, Christiani D, Zhang J, et al. Body Mass Index (BMI), BMI change, and overall survival in patients with SCLC and NSCLC: a pooled analysis of the international lung cancer consortium. J Thorac Oncol. 2019;14(9):1594-1607.

32. Chen LK, Woo J, Assantachai P, Auyeung TW, Chou MY, Iijima K, Jang HC, et al. Asian Working Group for Sarcopenia: 2019 consensus update on sarcopenia diagnosis and treatment. J Am Med Dir Assoc. 2020;21(3):300-307 e302. 